Population Study

Poster

Abstract ID: 33

\title{
Oral health knowledge of periodontal disease among final year non-dental students of IIUM Kuantan Campus
}

Basma Ezzat Mustafa | Noorlide Abu Kasim | Lina Hilal Al-Bayati | Fatin Afiqah Zainudin | Najwa Amani Abu Osman

Kulliyyah of Dentistry, International Islamic University Malaysia

Introduction: To determine the levels of oral health knowledge of periodontal disease and its determinants among final year non-dental students of IIUM Kuantan Campus. Methods: A cross-sectional study was conducted among 284 final year non-dental students from five different kulliyyah using a validated structured questionnaire. The questionnaire consisted of items to assess participants' demographic and socioeconomic variables, dental health education, periodontal disease knowledge, and oral hygiene practice. The relationship between levels of knowledge with the socioeconomic background, oral hygiene practice, and dental health education were analyzed using Chi-square Test and One-way ANOVA. Statistical analysis was based on probability values of less than 0.05 $(p<0.05)$. Results: $70.4 \%(200 / 284)$ of the participants were female and $29.6 \%(84 / 284)$ were male with mean age 23.5. Limited knowledge was seen more in non-medical students than medical students significantly $(p<0.05)$ in periodontal disease factors $(43.8 \%$ vs $21.8 \%)$, relationship with systemic disease $(50.5 \%$ vs $29.6 \%)$, signs $(28.6 \%$ vs $11.7 \%)$, symptoms $(23.8 \%$ vs $10.1 \%)$, and prevention $(26.7 \%$ vs $12.3 \%)$ whereas causes $(34.3 \%$ vs $32.4 \%)$ and treatment $(62.9 \%$ vs $58.1 \%)$ were not significantly different $(p>0.05)$. Therefore, medical students had higher knowledge of periodontal disease $(p<0.05)$ than those from non-medical students $(26.8 \%$ vs $16.2 \%)$. There were no relationships between knowledge levels with other determinants. Conclusions: There were significant differences in level of periodontal disease knowledge except causes and treatment between medical and non-medical students. Thus, periodontal health knowledge education promotion should be emphasized among non-medical students. Further study should be carried out to improve oral hygiene practices among students.

KEYWORDS: knowledge, periodontal disease, non-dental students 